\title{
THE TWO COMPONENT MAGNETIC FIELD OF THE GALAXY
}

\author{
R.R. ANDREASSIAN ${ }^{1}$, A.N. MAKAROV 1,2 \\ 1. Byurakan Astrophysical Observatory, \\ 378433 Armenia, USSR \\ 2. Buryat Pedagogical Institute, \\ 670000 Ulan-Ude, Buryatia, USSR
}

The present paper is devoted to a study of the magnetic field configuration of our Galaxy based on Faraday rotation measures (RM) of 185 pulsars and 802 extragalactic radio sources. RM data of pulsars located near the plane of the Galaxy are used for the study of magnetic fields in neighbouring spiral arms. For the distribution of spiral arms the well-known model of Georgelin and Georgelin (1976) is used. The calculations show (for details see Andreassian and Makarov, 1987, 1989) that in the Perseus spiral arm and the local Orion arm the magnetic fields have approximately the same directions $\left(\ell_{0} ; b_{0}\right) \approx\left(80^{\circ} ; 0^{\circ}\right)$, while in the Sagittarius-Carina arm the magnetic field has an opposite direction.

RM data of extragalactic radio sources are used for construction of the picture of average RM distribution in Galactic coordinates (Andreassian and Makarov, 1987). This distribution gives the direction of the magnetic field. For the southern hemisphere the magnetic field has the same direction as the local Orion arm. In the northern hemisphere there exist two regions with oppositely directed magnetic fields. For explanation of the RM distribution we offer a new two-component magnetic field model of the Galaxy. According to this model besides the spiral arm magnetic fields (plane component) there exists a 'halo' component of the magnetic field which stretches far from the Galactic plane and completely surrounds the plane component. The RM distribution is well explained if we suppose that the magnetic field 'halos' in the southern and northern hemispheres are oppositely directed. The suggested model of 'halo' magnetic field is verified and confirmed by data from 54 pulsars located at large distances from the Galactic plane ( $h>400 \mathrm{pc}$ ).

\section{References}

Andreassian, R.R. and Makarov, A.N. (1987) Astrofizika 28, 419. Andreassian, R.R. and Makarov, A.N. (1989) Astrofizika 30, 170. Georgelin, J.M. and Georgelin, J.P. (1976) Astron. Astrophys. 49, 57. 